\title{
Is methicillin-resistant Staphylococcus Aureus
} infection associated with higher mortality and morbidity in hospitalized patients? A cohort study
of 55 I patients from South Western India

This article was published in the following Dove Press journal: Risk Management and Healthcare Policy

\author{
Aparajita Chatterjee' \\ Shipra Rai' \\ Vasudeva Guddattu \\ Chiranjay Mukhopadhyay ${ }^{3}$ \\ Kavitha Saravu'1,4 \\ 'Department of Medicine, Kasturba \\ Medical College, Manipal Academy of \\ Higher Education (MAHE), Manipal, \\ Madhav Nagar, Karnataka, India; \\ ${ }^{2}$ Department of Statistics, Prasanna \\ School of Public Health, Manipal \\ Academy of Higher Education \\ (MAHE), Manipal, Madhav Nagar, \\ Karnataka, India; ${ }^{3}$ Department of \\ Microbiology, Kasturba Medical \\ College Manipal Academy of Higher \\ Education (MAHE), Manipal, Madhav \\ Nagar, Karnataka, India; ${ }^{4}$ Manipal \\ McGill Center for Infectious Diseases, \\ Prasanna School of Public Health, \\ Manipal Academy of Higher Education \\ (MAHE), Manipal, Madhav Nagar, \\ Karnataka, India
}

\section{Correspondence: Shipra Rai} Department of Medicine, Kasturba Medical College, Manipal Academy of Higher Education (MAHE), Manipal, Madhav Nagar, Karnataka 576104, India Tel +91 991 6809269

Fax +91 8202571934

Email dr.shiprarai@gmail.com

Kavitha Saravu

Department of Medicine, Kasturba Medical College, Manipal Academy of Higher Education, Manipal, Madhav Nagar, Karnataka, India,

Tel +9l 9448107636

Fax +9| 8202571934

Email kavithasaravu@gmail.com
Purpose: To determine morbidity and mortality of methicillin-resistant Staphylococcus aureus (MRSA) and methicillin-sensitive $S$. aureus (MSSA) infections in a tertiary health care facility. Methods: A cohort study among hospitalized adult patients with culture proven MRSA or MSSA monoinfection were recruited in a tertiary referral center in South India from November 2011 to December 2012.

Results: Of total 551 subjects, 284 (52\%) had MRSA and 267 (48\%) MSSA infection. A total of 184 (65\%) subjects had health care-associated MRSA (HA-MRSA) and 100 (35\%) community-associated MRSA (CA-MRSA). Chronic kidney disease and recent antibiotic use had significant association with MRSA. MRSA patients had significant respiratory infection (OR $2.24[1.04,5.16])$ and bacteremia (OR $2.24[10.40,5.16])$, relative to MSSA. MSSA group had better survival function compared to MRSA group $(P=0.028)$. Median duration of ICU stays were 5 days (IQR 4, 8) and 2 days (IQR 2,2) in MRSA and MSSA, respectively. Complications such as acute kidney injury, sepsis, multiorgan dysfunction, need for supportive measures were more in the MRSA group.

Conclusion: MRSA imposes a huge burden in Indian scenario and HA-MRSA remains the main culprit. Patients with history of chronic kidney disease and recent use of antibiotics were found to be at a higher risk. Patients with MRSA infections tend to have poorer outcomes in terms of longer hospital stay, greater complications, and mortality.

Keywords: methicillin-resistant Staphylococcus aureus, Staphylococcus aureus, risk factors, outcome, mortality, India, prognosis, impact, morbidity

\section{Introduction}

Methicillin-resistant Staphylococcus aureus (MRSA) is one of the most commonly implicated agent in nosocomial infections in the US intensive care units. It accounts for more than $60 \%$ of all intensive care unit and is one of the leading causes of surgical site infections in tertiary care hospitals in North America. ${ }^{1-4}$ MRSA is a prominent emerging pathogen in the Indian subcontinent, with incidence varying from $25 \%$ in western part to $50 \%$ in southern part. ${ }^{5,6} \mathrm{~A}$ multicentric study conducted across 15 tertiary care centers in India from 2008 to 2009 showed overall prevalence of MRSA infection to be $41 \%$ among $S$. aureus isolates.

Over the last decade, there has been an upsurge of community-associated MRSA (CA-MRSA) worldwide. ${ }^{8}$ CA-MRSA is one of the leading causes of skin and soft tissue infections in the USA. ${ }^{9}$ Moreover, CA-MRSA is also encroaching on nosocomial settings. ${ }^{10}$ Furthermore, infections with resistant organisms are notorious to impart 
significant health and economic consequences. While initial studies that investigated the impact of MRSA on mortality had produced conflicting results, more recent ones have shown increased mortality associated with MRSA infections in various settings. ${ }^{11,12}$ Although several of these studies have evaluated the difference in mortality between patients with MRSA and methicillin-sensitive $S$. aureus (MSSA), less is known about the effect of MRSA on other patient outcomes, such as length of hospital stay, length of antibiotics prescribed, and need for supportive measures. ${ }^{13}$

In this landscape of changing epidemiology of MRSA infections worldwide and dearth of literature on risk factors and outcomes of MRSA infections from the Indian subcontinent, a study addressing this knowledge gap was obligatory. The aims of our study were twofold. The first was to determine the clinical characteristics and risk factors of MRSA and MSSA infections in our tertiary care hospital. The second was to determine the outcomes of patients with MRSA and MSSA infections.

\section{Methods Study design}

We conducted a cohort study to compare the risk factors and outcomes of MRSA and MSSA infections among hospitalized adult patients in a 2030-bed tertiary referral center in Manipal, South India from November 2011 to December 2012. Study group consisted of individuals $\geq 18$ years with culture proven MRSA or MSSA monoinfection, where the organism was isolated from aspirated pus, body fluids, or blood. Only the first $S$. aureus infection for the individual patient was included. All samples were collected under aseptic conditions following recommended procedures. Individuals with cultures growing any other organism simultaneously along with MRSA/MSSA were excluded. Out of 612 patients with $S$. aureus infections during the study period, 551 patients fulfilled the inclusion criteria and were analyzed further (Figure 1).

\section{Variable definitions}

A case (MRSA/MSSA) was defined as a hospitalized patient who had at least one positive culture for MRSA or MSSA. It was considered community-associated (CA-MRSA) when it appeared within 48 hours of admission without any health care risk factors (the presence of invasive device, history of surgery, hospitalization, dialysis, or residence in a long-term care facility within the preceding 12 months) or health careassociated (HA-MRSA) with onset $\geq 48$ hours after admission or with $\geq 1$ health care risk factor. ${ }^{14}$ Infective endocarditis was defined as per Dukes criteria. ${ }^{15}$ Acute kidney injury (AKI) was defined by a rise of at least $0.3 \mathrm{mg} / \mathrm{dL}$ of creatinine or $50 \%$ higher than baseline within a 24 - to 48 -hour period. ${ }^{15}$ Gangrene was defined as gray/blackish discoloration of body part/limb with or without line of demarcation. Multiorgan dysfunction syndrome was defined as dysfunction of more than one organ, requiring intervention to maintain homeostasis. ${ }^{15}$ Septic shock was defined as arterial blood pressure $<90 \mathrm{mmHg}$ systolic, or $40 \mathrm{mmHg}$ less than patient's normal blood pressure for at least 1 hour despite adequate fluid resuscitation or need for vasopressors to maintain systolic blood pressure $90 \mathrm{mmHg}$ or mean arterial pressure $70 \mathrm{mmHg}$. ${ }^{15}$ Acute respiratory distress syndrome was defined as $\mathrm{PaO}_{2} / \mathrm{FiO}_{2}$ $<200 \mathrm{mmHg}$ with bilateral alveolar or interstitial infiltrates and no clinical evidence of increased left atrial pressure. ${ }^{15}$ Systemic inflammatory response syndrome was defined by two or more of the following conditions, if fulfilled-fever (oral temperature $>38^{\circ} \mathrm{C}$ ) or hypothermia $\left(<36^{\circ} \mathrm{C}\right)$, tachypnea ( $>24$ breaths/min), tachycardia (heart rate $>90$ beats $/ \mathrm{min}$ ) and leukocytosis $(>12,000 / \mathrm{L})$, leucopenia $(<4,000 / \mathrm{L})$ or $>10 \%$ bands. ${ }^{15}$ Recent antibiotic use was defined as use of antibiotics for $\geq 7$ days within the preceding 2 months. Adverse outcome was defined as a composite of death and discharge from the hospital against medical advice in a critical condition.

\section{Data collection}

All the isolates were identified as MRSA or MSSA based on the culture sensitivity reports. Blood culture was done using BacT-ALERT 3D automated microbial detection system (bioMérieux, Marcy l'Etoile, France), and growth of S. aureus was identified according to standard laboratory procedures. Antimicrobial susceptibility was tested in accordance with Clinical and Laboratory Standards Institute guidelines by Kirby-Bauer disk diffusion method. ${ }^{16}$ Cefoxitin disk diffusion was used to categorize $S$. aureus isolates as MRSA or MSSA. Once the subject was identified data including the demographic details, type of infection, risk factors, complications, treatment given, and outcomes were collected in a standard proforma after obtaining written informed consent from the patient or his/her relatives. Patients were initiated with empiric antibiotic therapy followed by specific therapy as per drug susceptibility reports along with surgical interventions wherever indicated, as per the treating clinicians' judgment. All patients were followed up till discharge from the hospital or death.

\section{Statistical analyses}

Categorical variables are summarized by frequency and percentage. Continuous variables are summarized using mean 


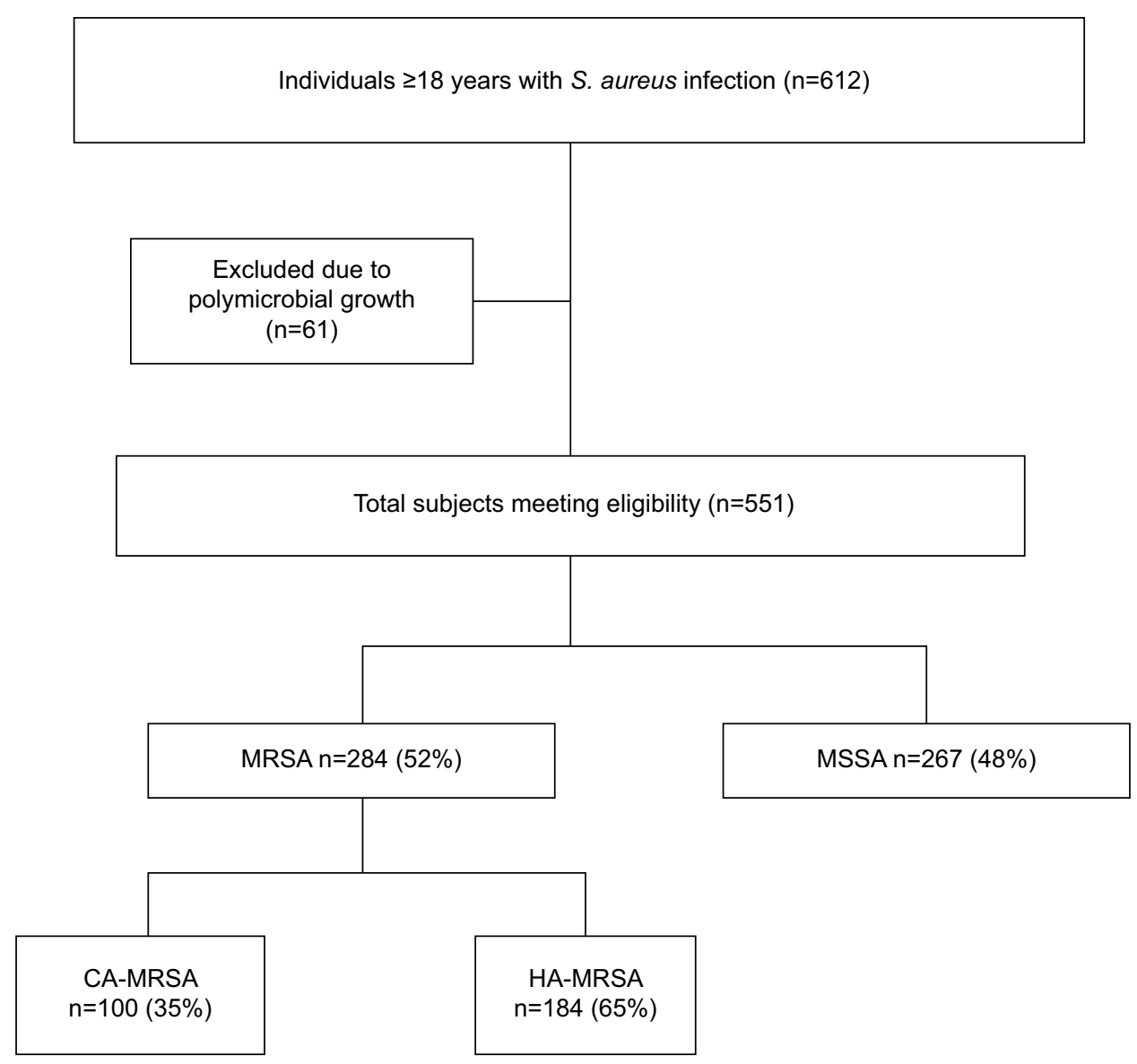

Figure I Flowchart depicting patient enrollment.

Abbreviations: HA-MRSA, health care-associated MRSA; MRSA, methicillin-resistant Staphylococcus aureus; MSSA, methicillin-sensitive S. aureus.

and SD (for normally distributed variables) or median and IQR (for non-normally distributed variables). Chi-squared test was used as test of association between two categorical variables whereas OR with $95 \%$ CI was used to report the strength of association between them. Mann-Whitney $U$-test was used to compare medians across binary variable. Log rank test was used to compare survival function between two groups. All the data were analyzed using SPSS 15 and Stata 13 . A $P$-value $<0.05$ was considered statistically significant.

\section{Ethical approval/statement}

The study was approved by Kasturba Medical College and Kasturba Hospital Ethics committee. Written informed consent was obtained from all participants. Patients' data were anonymized to maintain confidentiality.

\section{Results}

\section{Profile of MRSA and MSSA infections}

Out of 551 subjects, $284(52 \%)$ individuals were infected with MRSA, whereas 267 (48\%) had MSSA infection. A 184
(65\%) of the subjects were classified as having HA-MRSA as compared to the 100 (35\%) who had CA-MRSA infections (Figure 1). Of the MRSA patients who were classified as having HA-MRSA infection, 162 (88\%) had hospitalization within 12 months, 86 (47\%) of them grew MRSA from cultures drawn 48 hours after admission, 102 (55\%) had surgery in the past 12 months, $18(10 \%)$ had a percutaneous device in situ, and $15(8 \%)$ patients had undergone hemodialysis in the last 12 months.

Mean age of the MRSA group was $46.39 \pm 16.08$ and in MSSA was $44.77 \pm 14.31$. The number of males in MRSA and MSSA groups were 197 (70\%) and 175 (66\%), respectively. The distribution of participants across age group and gender was homogeneous between the two groups.

Spectrum of infections produced by MRSA/MSSA are summarized in Table 1. Skin and soft tissue infections predominated the clinical presentations in both the groups without any difference. However, MRSA was associated with significantly higher odds of respiratory infections (OR 2.24 [1.04, 5.16]) and bacteremia (OR 2.24 [10.40, 5.16]) than MSSA and had inverse association with deep 
abscesses (OR $0.51[0.31,0.81])$ and septic arthritis (OR $0.30[0.08,0.89])$.

\section{Distribution of clinical characteristics of MRSA and MSSA infections}

The distribution of risk factors and comorbidities associated with infections are depicted in Table 2. Chronic kidney disease (OR 7.60 [2.90, 25.25]) and recent use of antibiotics (OR $1.74[1.13,2.68])$ showed significant association with MRSA infection as compared to MSSA.

\section{Treatment of MRSA and MSSA infections}

In both the groups, $96 \%$ of the patients were empirically started on antimicrobial therapy. Notably, in the MRSA group, only 83/284 (29\%) patients received empirical antibiotics that were effective against MRSA, as compared to 215/267 (80\%) of MSSA patients who received antibiotics that the organism was sensitive to. The most commonly used empirical antibiotic was amoxicillin-clavulanic acid in both the MRSA (151/284 [53\%]) and MSSA (148/267 [55\%]) groups. Subsequent to culture reports, while the empiric antibiotic treatment was continued in 137/267 (51\%) of MSSA patients, antibiotics were changed in 212/284 (75\%) of MRSA patients. As specific anti-MRSA regimen, linezolid was initiated in $145 / 284(51 \%)$, whereas $36 / 284$ $(13 \%)$ were given co-trimoxazole, 17/284 (6\%) received vancomycin, and 14/284 (5\%) received teicoplanin. Moreover, 33/267 (12\%) of the MSSA patients were changed over to clindamycin, $32 / 267$ (12\%) to co-trimoxazole, and $21 / 267$ (8\%) to linezolid. Surgical debridement and removal of focus of infection were required in 204/267 (76\%) of the MSSA patients as compared to $180 / 284(63 \%)$ of MRSA patients.

\section{Distribution of complications and outcomes of MRSA vs MSSA infections}

The distribution of complications, supportive measures, durations of ICU stay, hospital stay, and antibiotic prescription in MRSA and MSSA group is presented in Tables 3 and 4 . The occurrence of complications such

Table I Distribution of sites of MRSA and MSSA infections

\begin{tabular}{|c|c|c|c|}
\hline Site of infection ${ }^{a}$ & $\begin{array}{l}\text { MRSA (N=284) } \\
\text { n (\%) }\end{array}$ & $\begin{array}{l}\text { MSSA }(\mathrm{N}=267) \\
\mathrm{n}(\%)\end{array}$ & OR $(95 \% \mathrm{Cl})$ \\
\hline Skin and soft tissue infections & $142(39)$ & $124(39)$ & $0.99(0.69, \mathrm{I} .4 \mathrm{I})$ \\
\hline Deep abscess & $38(13)$ & $62(23)$ & $0.5 \mathrm{I}(0.3 \mathrm{I}, 0.8 \mathrm{I})$ \\
\hline Surgical site & $32(\mathrm{II})$ & $20(7)$ & $1.56(0.84,2.97)$ \\
\hline Abdominal sepsis & $2(1)$ & $2(1)$ & $0.93(0.06,13.05)$ \\
\hline Osteomyelitis & $23(8)$ & $26(10)$ & $0.81(0.43,1.53)$ \\
\hline Septic arthritis & $5(2)$ & $15(6)$ & $0.30(0.08,0.89)$ \\
\hline Respiratory tract infection & $25(9)$ & II (4) & $2.24(1.04,5.16)$ \\
\hline Bacteremia & $25(9)$ & II (4) & $2.24(10.40,5.16)$ \\
\hline
\end{tabular}

Notes: ${ }^{D}$ ifferent subgroups are not mutually exclusive.

Abbreviations: MRSA, methicillin-resistant Staphylococcus aureus; MSSA, methicillin-sensitive S. aureus.

Table 2 Distribution of risk factors and comorbidities associated with MRSA and MSSA infections

\begin{tabular}{|l|l|l|l|}
\hline Variables & $\begin{array}{l}\text { MRSA (N=284) } \\
\mathbf{n}(\%)\end{array}$ & $\begin{array}{l}\text { MSSA (N=267) } \\
\mathbf{n}(\%)\end{array}$ & OR (95\% CI) \\
\hline Diabetes mellitus & $108(38)$ & $82(31)$ & $1.38(0.96,2.03)$ \\
\hline CKD & $36(13)$ & $5(2)$ & $7.60(2.90,25.15)$ \\
\hline Recent use of antibiotics & $77(27)$ & $47(18)$ & $1.74(I .13,2.68)$ \\
\hline Prosthetics & $32(I)$ & $35(13)$ & $0.84(0.48,1.44)$ \\
\hline Immunosuppressant use & $20(7)$ & $17(6)$ & $1.14(0.54,2.32)$ \\
\hline Malignancy & $5(2)$ & $8(3)$ & $0.58(0.15,2.04)$ \\
\hline Alcoholism & $16(6)$ & $10(4)$ & $1.53(0.64,3.85)$ \\
\hline Heart disease & $24(9)$ & $28(10)$ & $0.78(0.42,1.45)$ \\
\hline CVA & $7(2)$ & $8(3)$ & $0.81(0.24,2.62)$ \\
\hline COPD & $7(2)$ & $5(2)$ & $1.32(0.35,5.35)$ \\
\hline Rheumatoid arthritis & $4(1)$ & $2(I)$ & $1.89(0.27,21.06)$ \\
\hline SLE & $5(2)$ & $2(I)$ & $2.37(0.38,25.09)$ \\
\hline HIV & $5(2)$ & $2(1)$ & $2.37(0.38,25.09)$ \\
\hline
\end{tabular}

Abbreviations: CKD, chronic kidney disease; CVA, cerebrovascular accident; HD, hemodialysis; MRSA, methicillin-resistant Staphylococcus aureus; MSSA, methicillinsensitive S. aureus; SLE, systemic lupus erythematosus. 
as sepsis, AKI, and multiorgan dysfunction were significantly higher in the MRSA group as compared to MSSA group (Table 3). The median (IQR) duration of hospital stay in the MRSA group was 14 days (8.0-18.75 days) as compared to 8 days (4-16 days) in the MSSA group. The total duration of hospital stay was significantly longer in the MRSA group $(P<0.001)$ (Table 4). A total of 112 $(40 \%)$ of MRSA patients required ICU care in contrast to 38 (14\%) of MSSA patients. Among patients requiring ICU care, the median IQR duration of ICU stays were 5 days $(4,8)$ and 2 days $(2,2)$ in MRSA and MSSA, respectively, which was statistically significant $(P<0.001)$. The median (IQR) duration of antibiotics received in MRSA was 20 days (14-27 days); in MSSA, it was 14 days (1024 days). Overall, the duration of antibiotic therapy was significantly longer in MRSA patients compared to MSSA patients. Likewise, the need for supportive measures were significantly higher in MRSA patients compared to MSSA patients (Table 4).

Case fatality rates were $7(2 \%)$ and $3(1 \%)$ for MRSA and MSSA infections, respectively; $18(6 \%)$ MRSA patients took discharge against medical advice (worse at discharge) in view of poor prognosis and lack of affordability of further health care as compared to $6(2 \%)$ of MSSA patients. Of the 34 patients who had an adverse outcome, 24 patients had received inappropriate empirical antibiotics. Inappropriate empiric antibiotic therapy had significant $(P=0.006)$ association with adverse outcome. The mean survival time for MSSA group is significantly higher as compared to MRSA group.

\section{Discussion}

The present study aimed to determine the distribution of risk factors and outcomes of MRSA infection in an Indian tertiary health care facility. A high proportion (52\%) of MRSA infections was in concordance with other studies from India and the USA., ${ }^{2,7}$ In Europe, however, the prevalence of MRSA varies considerably, ranging from $0.4 \%$ in Sweden to $48.4 \%$ in Belgium. ${ }^{17}$ We further classified the MRSA patients as health care-associated MRSA (HAMRSA) or community-associated MRSA (CA-MRSA). In the MRSA sub-group, 184 (65\%) HA-MRSA. These findings are consistent with other studies conducted in India and elsewhere. ${ }^{7}$ However, this is in contrast to a study

Table 3 Distribution of complications in MRSA vs MSSA infections

\begin{tabular}{|l|l|l|l|}
\hline Complication & $\begin{array}{l}\text { MRSA (N=284) } \\
\mathbf{n}(\%)\end{array}$ & $\begin{array}{l}\text { MSSA (N=267) } \\
\mathbf{n}(\%)\end{array}$ & OR (95\% CI) \\
\hline SIRS & $4 I(14)$ & $14(5)$ & $3.05(1.58,6.20)$ \\
\hline Shock & $23(8)$ & $18(7)$ & $1.22(0.61,2.45)$ \\
\hline Acute kidney injury & $26(9)$ & $9(3)$ & $2.88(1.28,7.13)$ \\
\hline ARDS & $17(4)$ & $15(3)$ & $1.07(0.49,2.35)$ \\
\hline Gangrene & $27(10)$ & $33(12)$ & $0.74(0.42,1.32)$ \\
\hline MODS & $26(9)$ & $9(3)$ & $2.88(1.28,7.13)$ \\
\hline
\end{tabular}

Abbreviations: ARDS, acute respiratory distress syndrome; MRSA, methicillin-resistant Staphylococcus aureus; MSSA, methicillin-sensitive S. aureus; MODS, multiorgan dysfunction; SIRS, systemic inflammatory response syndrome.

Table 4 Distribution of supportive measures, durations of ICU stay, hospital stay, and antibiotic prescription with MRSA vs MSSA infection

\begin{tabular}{|c|c|c|c|c|}
\hline Variables & Duration/characteristic & $\begin{array}{l}\text { MRSA } \\
(\mathrm{N}=284) \\
n(\%) \\
\end{array}$ & $\begin{array}{l}\text { MSSA } \\
(N=267) \\
n(\%)\end{array}$ & $P$-value \\
\hline \multirow[t]{3}{*}{ Hospitalization period } & $<7$ days & $50(18)$ & $100(37)$ & \\
\hline & $7-21$ days & $194(68)$ & $126(47)$ & \\
\hline & $>21$ days & $40(14)$ & $4 I(16)$ & $<0.001$ \\
\hline \multirow[t]{3}{*}{ ICU stay } & None & $172(61)$ & $229(86)$ & \\
\hline & $<7$ days & $72(25)$ & $37(14)$ & \\
\hline & $\geq 7$ days & $40(14)$ & $I(0)$ & $<0.001$ \\
\hline \multirow[t]{3}{*}{ Duration of antibiotics } & $<7$ days & $10(4)$ & $45(17)$ & \\
\hline & $7-21$ days & $187(66)$ & $152(57)$ & \\
\hline & $>21$ days & $87(30)$ & $70(26)$ & $<0.001$ \\
\hline \multirow[t]{4}{*}{ Supportive measures } & Inotropes & $18(6)$ & $15(6)$ & \\
\hline & Blood products & $28(10)$ & $18(7)$ & \\
\hline & Dialysis & $22(8)$ & $I I(4 I)$ & \\
\hline & Mechanical ventilation & $15(5)$ & $9(2)$ & $<0.001$ \\
\hline
\end{tabular}

Abbreviations: MRSA, methicillin-resistant Staphylococcus aureus; MSSA, methicillin-sensitive S. aureus. 
from South India that had reported a prevalence of $74 \%$ of CA-MRSA. ${ }^{18}$ This difference was probably due to the lone criterion used for CA-MRSA in their study with all positive cultures drawn within 48 hours of hospitalization being taken as community acquired.

Skin and soft tissue infections predominated in both the groups, proportions being 46\% for MSSA and 49\% for MRSA, in concurrence with other studies. ${ }^{7}$ However, MRSA was associated with significantly higher odds of respiratory infections and bacteremia (OR 2.24 [95\% CI $1.04,5.16]$ ), which reiterate the findings of previous studies. ${ }^{19}$ Notably, there was no case of infective endocarditis due to $S$. aureus.

Risk factors found to be statistically significant for MRSA were chronic kidney disease and recent use of antibiotics. ${ }^{20}$ Prior receipt of antibiotics is an established risk factor that selects for drug-resistant MRSA and facilitates colonization and infection. However, we could not assign a particular class of antibiotics; the data could not be ascertained with certainty in all patients as available prescription prior to the hospitalization was uniformly poor in the study setting. Older age, diabetes mellitus, and chronic kidney disease were significantly associated with MRSA surgical site infections compared to MSSA. ${ }^{21}$ In another study among 13,796 adult ICU patients, MRSA was associated with cancer and chronic renal failure. ${ }^{22}$ Remarkably, in the present study, no patient had history of intravenous drug abuse.

There was a significant increase in survival function of MSSA group as compared to MRSA group $(P=0.028)$ (Figure 2). These results are in conformity with those observed by Hanberger et al in critically ill patients and Engemann et al among surgical site infection patients..$^{21,22}$ Moreover, in critically ill patients, MRSA bacteremia was found to have a higher attributable mortality than MSSA bacteremia after adjusting for disease severity and acute illness and additionally a meta-analysis has ascertained higher mortality with MRSA bacteremia compared to MSSA bacteremia. ${ }^{11,12}$ The number of hospital days, the need and duration of ICU stay, and the total duration of antibiotics prescription were also longer for MRSA patients than MSSA patients, as observed by others. ${ }^{22}$ The above-noted findings can be expounded on the basis that MRSA patients had more invasive infections, had more co-morbidities and complications as compared to the MSSA sub-group, and they tended to receive appropriate antibiotic therapy at a later date, all delaying their recovery.

There has been no evidence hitherto to suggest that MRSA strains are more virulent than MSSA strains. ${ }^{12}$ However, MRSA-associated increased mortality is accounted for by SCCmec type II in a study that adjusted for source

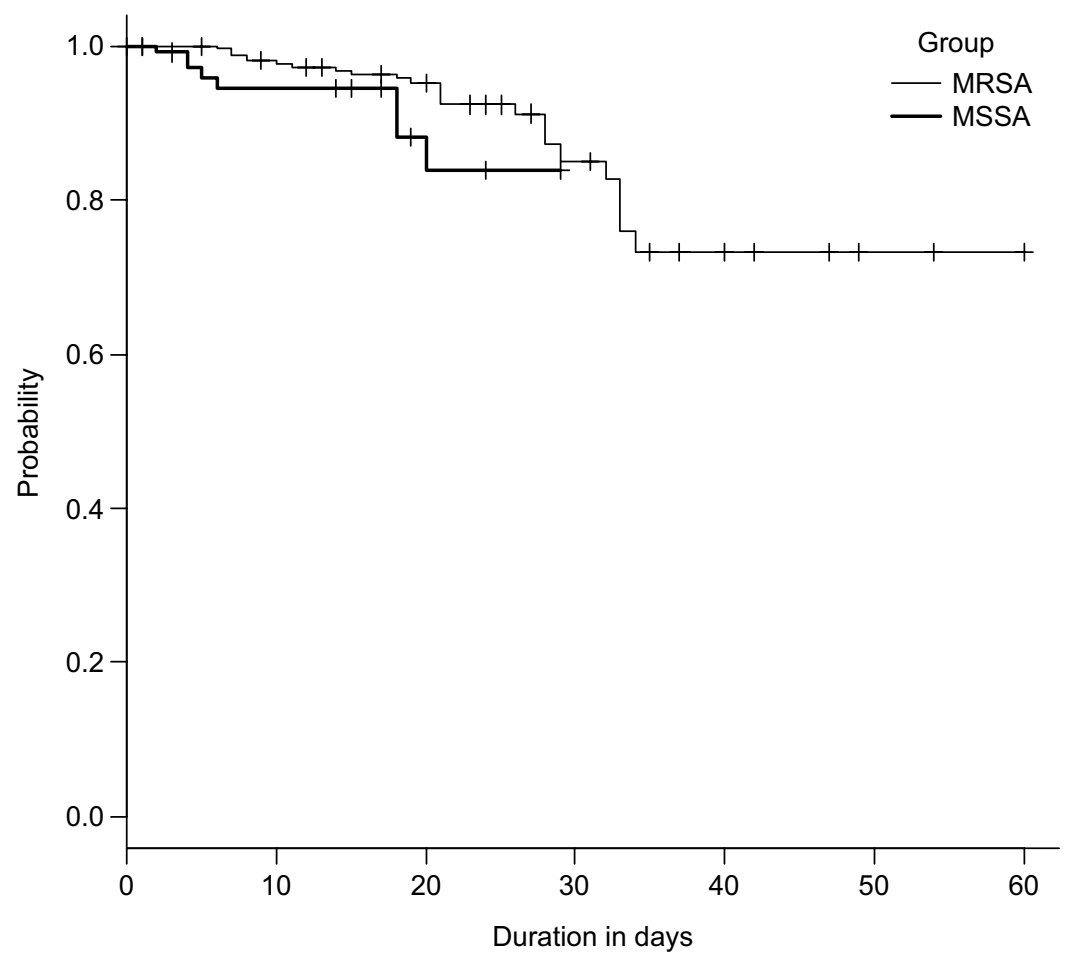

Figure 2 Kaplan-Meier graph depicting survival function across the groups.

Abbreviations: MRSA, methicillin-resistant Staphylococcus aureus; MSSA, methicillin-sensitive S. aureus. 
of infection, comorbid conditions, illness severity, and treatment type. ${ }^{23}$ A notable feature in the present study was that only $29 \%$ of MRSA patients received antibiotics that the organism was sensitive to, whereas the vast majority of MSSA patients (81\%) received appropriate empirical antibiotics. Similar findings were also noted by Soriano et al and Eshwara et al in their studies. ${ }^{18,24}$ Only one-third of MRSA patients had received appropriate empiric treatment and three-fourth of MRSA patients received a change in prescription post cultures. Notably, failure to receive early appropriate antibiotic in MRSA group was a major contributing factor for increased mortality and supportive requirements. A delay in receiving effective antibiotic therapy was associated significantly with all cause and attributable mortality in a study from Thailand. ${ }^{25}$

The present study has significant strengths. To the best of our knowledge, this is one of the largest clinical studies on MRSA infection in a non-outbreak setting from India, which prospectively recruited patients across all departments and thus gives us an idea about the wide spectrum of infections that can be caused by this versatile microbe. This study has sought to identify the risk factors and has used standard definition of CA-MRSA. In addition to mortality, the study has also addressed lesser studied outcomes such as length of hospital stay, ICU requirement, need for supportive care, and duration of antibiotics prescribed giving a useful surrogate metric of resource utilization and economic consequences. Outcomes of infections with susceptible vs resistant organisms is affected by the severity of underlying illness, as this by itself may influence the length of hospitalization and mortality/morbidity profiles. In the present study, as we did not adjust for underlying diseases or severity of clinical condition, there is a possibility that the association of MRSA with mortality and adverse outcomes has been overestimated. The standard scoring systems that are in use for ICU patients were not applicable for all our patients with $S$. aureus infections across the departments and hence could not be used. Although MSSA can be hospital or community acquired, this distinction was not assigned in the present study and hence could not be compared. Definition of sepsis and septic shock has changed since the study; however, this would not change the results of the present study.

Rates of nasal carriage of MRSA have been studied by various investigators in India, and they range from $7.7 \%$ in school children, $3 \%-10 \%$ in predominantly outpatient population to $7.5 \%-25 \%$ in health care workers, highlighting the potential role of health care workers in the transmission. ${ }^{26-29}$ However, the present study lacks MRSA carrier status estimation, molecular characterization, and panton-valentine leukocidin toxin carriage status. Future multicentric studies that adequately adjust for the severity of underlying illness and treatment instituted are needed to better clarify the risk factors for mortality and improve the treatment outcomes.

\section{Conclusion}

More than half of all the staphylococcal infections in the present study were due to methicillin-resistant strains. Of these, the majority were health care associated. Risk factors associated with MRSA infections were chronic kidney disease and recent antibiotic use. These findings thus highlight the problem of MRSA in the Indian scenario. Our study reiterates that MRSA is associated with increased mortality and prolonged hospitalization. They were also more likely to have received inappropriate antibiotics at the onset. Hence, a greater degree of suspicion for MRSA in clinical setting, early detection of MRSA by rapid tests from blood stream infection by automated methods, along with timely prescription of appropriate antibiotics and source control are needed to improve the outcomes in these patients. Also, restricted use of antimicrobials, antibiotic stewardship, and infection control can help prevent further spread of these deadly multidrug-resistant organisms.

\section{Disclosure}

The authors report no conflicts of interest in this work.

\section{References}

1. National Nosocomial Infections Surveillance System. National Nosocomial Infections Surveillance (NNIS) System Report, data summary from January 1992 through June 2004, issued October 2004. Am J Infect Control. 2004;32(8):470.

2. Anderson DJ, Sexton DJ, Kanafani ZA, Auten G, Kaye KS. Severe surgical site infection in community hospitals: epidemiology, key procedures, and the changing prevalence of methicillin-resistant Staphylococcus aureus. Infect Control Hosp Epidemiol. 2007;28(9):1047-1053.

3. Hidron AI, Edwards JR, Patel J, et al; National Healthcare Safety Network Team; Participating National Healthcare Safety Network Facilities. NHSN annual update: antimicrobial-resistant pathogens associated with healthcare-associated infections: annual summary of data reported to the National Healthcare Safety Network at the Centers for Disease Control and Prevention, 2006-2007. Infect Control Hosp Epidemiol. 2008;29(11):996-1011.

4. Dukic VM, Lauderdale DS, Wilder J, Daum RS, David MZ. Epidemics of community-associated methicillin-resistant Staphylococcus aureus in the United States: a meta-analysis. PLoS One. 2013;8(1):e52722.

5. Patel AK, Patel KK, Patel KR, Shah S, Dileep P. Time trends in the epidemiology of microbial infections at a tertiary care center in west India over last 5 years. J Assoc Physicians India. 2010;58(Suppl): $37-40$.

6. Gopalakrishnan R, Sureshkumar D. Changing trends in antimicrobial susceptibility and hospital acquired infections over an 8 year period in a tertiary care hospital in relation to introduction of an infection control programme. J Assoc Physicians India. 2010;58(Suppl):25-31. 
7. Joshi S, Ray P, Manchanda V, Bajaj J, Chitnis D, Gautam V, Goswami P et al. Methicillin resistant Staphylococcus aureus (MRSA) in India: prevalence \& susceptibility pattern. Indian JMed Res. 2013;137(2):363.

8. Alvarez-Uria G, Reddy R. Prevalence and antibiotic susceptibility of community-associated methicillin-resistant Staphylococcus aureus in a rural area of India: is MRSA replacing methicillin-susceptible Staphylococcus aureus in the community? ISRN Dermatol. 2012;2012(21):1-5.

9. Moran GJ, Krishnadasan A, Gorwitz RJ, et al. Methicillin-resistant $S$. aureus infections among patients in the emergency department. $\mathrm{NEngl}$ J Med. 2006;355(7):666-674.

10. Popovich KJ, Weinstein RA, Hota B. Are community-associated methicillin-resistant Staphylococcus aureus (MRSA) strains replacing traditional nosocomial MRSA strains? Clin Infect Dis. 2008;46(6):787-794.

11. Blot SI, Vandewoude KH, Hoste EA, Colardyn FA. Outcome and attributable mortality in critically Ill patients with bacteremia involving methicillin-susceptible and methicillin-resistant Staphylococcus aureus. Arch Intern Med. 2002;162(19):2229-2235.

12. Cosgrove SE, Sakoulas G, Perencevich EN, Schwaber MJ, Karchmer AW, Carmeli Y. Comparison of mortality associated with methicillinresistant and methicillin-susceptible Staphylococcus aureus bacteremia: a meta-analysis. Clin Infect Dis. 2003;36(1):53-59.

13. Wang JL, Chen SY, Wang JT, et al. Comparison of both clinical features and mortality risk associated with bacteremia due to communityacquired methicillin-resistant Staphylococcus aureus and methicillinsusceptible $S$. aureus. Clin Infect Dis. 2008;46(6):799-806.

14. Klevens RM, Morrison MA, Nadle J, et al. Invasive methicillin-resistant Staphylococcus aureus infections in the United States. J Am Med Assoc. 2007;298(15):1763-1771.

15. Fauci AS, Braunwald E, Kasper DL, Hauser SL, Longo DL, Jameson JL. Harrison's Principles of Internal Medicine. 17th ed. New York: McGraw-Hill Education; 2008.

16. Clinical Laboratory Standards Institute. Performance Standards for Antimicrobial Disk Susceptibility Tests; Approved Standard. Wayne, PA: Laboratory Standard Institute; 2012.

17. Sader HS, Farrell DJ, Jones RN. Antimicrobial susceptibility of Grampositive cocci isolated from skin and skin-structure infections in European medical centres. Int J Antimicrob Agents. 2010;36(1):28-32.

18. Eshwara VK, Munim F, Tellapragada C, et al. Staphylococcus aureus bacteremia in an Indian tertiary care hospital: observational study on clinical epidemiology, resistance characteristics, and carriage of the Panton-Valentine leukocidin gene. Int J Infect Dis. 2013;17(11): e1051-e1055.
19. Filice GA, Nyman JA, Lexau C, et al. Excess costs and utilization associated with methicillin resistance for patients with Staphylococcus aureus infection. Infect Control Hosp Epidemiol. 2010;31(4):365-373.

20. Hershow RC, Khayr WF, Smith NL. A comparison of clinical virulence of nosocomially acquired methicillin-resistant and methicillin-sensitive Staphylococcus aureus infections in a university hospital. Infection Control. 1992;13(10):587-593.

21. Engemann JJ, Carmeli Y, Cosgrove SE, et al. Adverse clinical and economic outcomes attributable to methicillin resistance among patients with Staphylococcus aureus surgical site infection. Clin Infect Dis. 2003;36(5):592-598.

22. Hanberger $\mathrm{H}$, Walther $\mathrm{S}$, Leone $\mathrm{M}$, et al. Increased mortality associated with methicillin-resistant Staphylococcus aureus (MRSA) infection in the intensive care unit: results from the EPIC II study. Int J Antimicrob Agents. 2011;38(4):331-335.

23. Ganga R, Riederer K, Sharma M, et al. Role of SCCmec type in outcome of Staphylococcus aureus bacteremia in a single medical center. J Clin Microbiol. 2009;47(3):590-595.

24. Soriano A, Martínez JA, Mensa J, et al. Pathogenic significance of methicillin resistance for patients with Staphylococcus aureus bacteremia. Clin Infect Dis. 2000;30(2):368-373.

25. Nickerson EK, Wuthiekanun V, Wongsuvan G, et al. Factors predicting and reducing mortality in patients with invasive Staphylococcus aureus disease in a developing country. PLoS One. 2009;4(8):e6512.

26. Singh AK, Agarwal L, Kumar A, Sengupta C, Singh RP. Prevalence of nasal colonization of methicillin-resistant Staphylococcus aureus among schoolchildren of Barabanki district, Uttar Pradesh, India. $J$ Family Med Prim Care. 2018;7(1):162-166.

27. Sharma Y, Jain S, Singh H, Govil V. Staphylococcus aureus: screening for nasal carriers in a community setting with special reference to MRSA. Scientifica. 2014;2014(3):1-5.

28. Singh S, Malhotra R, Grover P, et al. Antimicrobial resistance profile of methicillin-resistant Staphylococcus aureus colonizing the anterior nares of health-care workers and outpatients attending the remotely located tertiary care hospital of North India. J Lab Physicians. 2017;9(4):317-321.

29. Patwardhan V, Kumar D, Goel V, Singh S, Sarman S. Changing prevalence and antibiotic drug resistance pattern of pathogens seen in community-acquired pediatric urinary tract infections at a tertiary care hospital of North India. J Lab Physicians. 2017;9(4):264-268.
Risk Management and Healthcare Policy

\section{Publish your work in this journal}

Risk Management and Healthcare Policy is an international, peer-reviewed open access journal focusing on all aspects of public health, policy, and preventative measures to promote good health and improve morbidity and mortality in the population. The journal welcomes submitted papers covering original research, basic science, clinical and epidemiological

\section{Dovepress}

studies, reviews and evaluations, guidelines, expert opinion and commentary, case reports and extended reports. The manuscript management system is completely online and includes a very quick and fair peerreview system, which is all easy to use. Visit http://www.dovepress.com/ testimonials.php to read real quotes from published authors. 